\title{
Compact radio cores in radio-quiet active galactic nuclei
}

\author{
A. Maini ${ }^{1,2,3,4}$, I. Prandoni ${ }^{3}$, R. P. Norris ${ }^{4,6}$, G. Giovannini ${ }^{1,3}$, and L. R. Spitler ${ }^{2,5}$ \\ 1 Dipartimento di Fisica e Astronomia, Università di Bologna, viale B. Pichat 6/2, 40127 Bologna, Italy \\ e-mail: alessandro.maini@studio.unibo.it \\ 2 Department of Physics and Astronomy, Macquarie University, Balaclava Road, North Ryde, 2109 NSW, Australia \\ 3 INAF-IRA, via P. Gobetti 101, 40129 Bologna, Italy \\ ${ }^{4}$ CSIRO Astronomy \& Space Science, PO Box 76, Epping, NSW 1710, Australia \\ 5 Australian Astronomical Observatories, PO Box 915, North Ryde, NSW 1670, Australia \\ 6 Western Sydney University, Locked Bag 1797, Penrith South, NSW 1797, Australia \\ Received 13 February 2016 / Accepted 18 March 2016
}

\begin{abstract}
Context. The mechanism of radio emission in radio-quiet (RQ) active galactic nuclei (AGNs) is still debated and might arise from the central AGN, from star formation activity in the host, or from either of these sources. A direct detection of compact and bright radio cores embedded in sources that are classified as RQ can unambiguously determine whether a central AGN significantly contributes to the radio emission.

Aims. We search for compact, high-surface-brightness radio cores in RQ AGNs that are caused unambiguously by AGN activity. Methods. We used the Australian Long Baseline Array to search for compact radio cores in four RQ AGNs located in the Extended Chandra Deep Field South (ECDFS). We also targeted four radio-loud (RL) AGNs as a control sample.

Results. We detected compact and bright radio cores in two AGNs that are classified as RQ and in one that is classified as RL. Two RL AGNs were not imaged because the quality of the observations was too poor.

Conclusions. We report on a first direct evidence of radio cores in RQ AGNs at cosmological redshifts. Our detections show that some of the sources that are classified as RQ contain an active AGN that can contribute significantly ( $\sim 50 \%$ or more) to the total radio emission.
\end{abstract}

Key words. galaxies: active - galaxies: high-redshift - radio continuum: galaxies

\section{Introduction}

Multi-wavelength studies of deep radio fields show that the sub-mJy population has a composite nature. While star-forming galaxies (SFG) dominate at $\mu$ Jy levels (e.g. Seymour et al. 2008), RL AGNs are the dominant component at flux densities $>0.5$ mJy (e.g. Mignano et al. 2008).

Recently, radio-quiet (RQ) active galactic nuclei (AGNs) have been shown to partly account for the flattening of the $1.4 \mathrm{GHz}$ source counts at flux densities $<0.5 \mathrm{mJy}$. RQ AGNs show AGN activity at non-radio wavelengths, but the origin of their radio emission is debated (Bonzini et al. 2013; Smolčić et al. 2015).

Most RQ AGNs are unresolved or barely resolved at a few arcsec scale, which is similar to the host galaxy size. RQ AGNs have also been found to share properties with SFGs. They have similar radio luminosities $\left(10^{22-24} \mathrm{~W} \mathrm{~Hz}^{-1}\right)$ and similar optical- or infrared-to-radio flux ratios. Their radio luminosity functions show similar evolutionary trends (Padovani 2011), and their host galaxies have similar colours, optical morphologies, and stellar masses (Bonzini et al. 2013). For all these reasons it was concluded that the radio emission in such RQ AGNs is mainly triggered by star formation (Padovani 2011; Bonzini et al. 2013).

Further support to this scenario was provided by Bonzini et al. (2015), who found that in RQ AGNs radioderived star formation rates (SFR) are consistent with FIR Herschel-derived SFR, though with a larger dispersion than for SFGs. This larger dispersion, on the other hand, does not rule out the possibility that some radio emission in these objects is associated with AGN activity.

It is well known that in the local Universe $(z \lesssim 0.5)$ both AGN and SF processes can contribute to the total radio emission in RQ AGNs (e.g. Seyfert 2 galaxies; Roy et al. 1998), and there is growing evidence that composite SF/AGN systems are common at medium to high redshift $(z \gtrsim 1-2$; see e.g. Daddi et al. 2007; Del Moro et al. 2013; Rees et al. 2016).

The most reliable way to separate AGN cores (size $\ll 1 \mathrm{kpc}$ ) from starbursts $(\gtrsim 1 \mathrm{kpc})$ in intermediate redshift $(z \sim 1)$ galaxies is through deep ( $\mu \mathrm{Jy}$-level) imaging at milli-arcsec (mas) resolution using Very Long Baseline Interferometry (VLBI).

The ECDFS is arguably the field in which the properties of sub-mJy RQ AGNs have been most extensively studied. Here we set constraints on the AGN contribution to the total radio emission in ECDFS sources classified as RQ AGNs using the Australian Long Baseline Array (LBA).

Throughout this Letter, we adopt a standard flat $\Lambda \mathrm{CDM}$ cosmology with $H_{0}=70 \mathrm{~km} \mathrm{~s}^{-1} \mathrm{Mpc}^{-1}$ and $\Omega_{\mathrm{M}}=0.30$.

\section{Strategy}

Our targets were selected from a $1.4 \mathrm{GHz}$ catalogue of $883 \mathrm{ra}$ dio sources obtained from VLA observations of the ECDFS field (Miller et al. 2013), which has a typical rms sensitivity of $\sim 7.4 \mu \mathrm{Jy}$ and an angular resolution of $2.8 \times 1.6 \operatorname{arcsec}^{2}$. The availability of extensive and deep multi-band information from radio (Huynh et al. 2012; Franzen et al. 2015) to X-ray band (Lehmer et al. 2005; Xue et al. 2011) allowed the classification of all the sources from Miller and collaborators into three classes: RL AGNs, RQ AGNs, and SFGs (Bonzini et al. 2013). 
A\&A 589, L3 (2016)

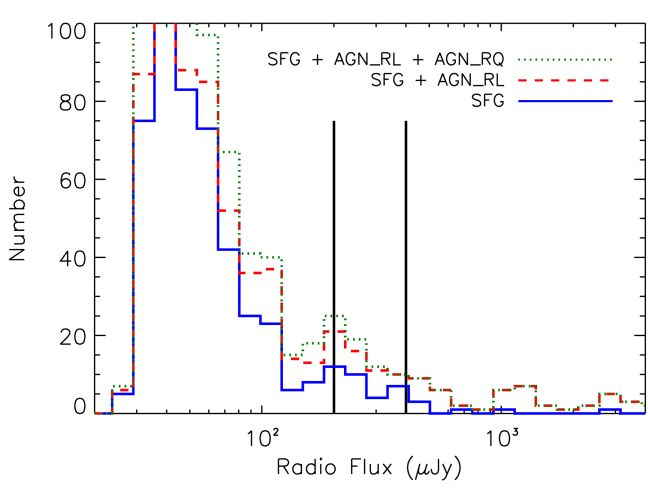

Fig. 1. Stacked histogram of the radio flux density distributions of the sources in Bonzini et al. (2013). The solid blue curve refers to SFGs, the red dashed curve adds to it the RL AGNs, the green dotted curve adds to them the secure $(\mathrm{QF}=3) \mathrm{RQ}$ AGNs. The two black vertical lines delimit the flux range of the sample we targeted (200-400 $\mu \mathrm{Jy})$.

The classification of Bonzini et al. (2013) is primarily based on the observed infrared-to-radio ratio $\left(q_{24 \mathrm{obs}}=\log \left(S_{24 \mu \mathrm{m}} / S_{1.4 \mathrm{GHz}}\right)\right)$. Any source that shows a significant radio excess with respect to an M82 template (redshifted up to $z=10$ ) is classified as RL. Sources with no significant radio excess are classified as RQ AGNs if they show clear evidence for AGN activity in the $\mathrm{X}$-rays $\left(L_{2-8 \mathrm{keV}}>10^{42} \mathrm{erg} \mathrm{s}^{-1}\right)$ or in the mid-infrared bands (i.e. the source lies inside the AGN wedge of the IRAC colour-colour diagram; Donley et al. 2012). A quality flag (QF) was also defined to express the reliability of the classification from secure $(\mathrm{QF}=3)$ to reasonable $(\mathrm{QF}=2)$ and tentative $(\mathrm{QF}=1)$ (Bonzini et al. 2013).

The ECDFS field has been observed with the VLBA by Middelberg et al. (2011). However, the position of the field $\left(\operatorname{Dec} \sim-28^{\circ}\right)$ is not well suited for the VLBA, and the average elevation during the experiment was only $\sim 20^{\circ}$. This limited the sensitivity of the observation: only sources brighter than $400-1000 \mu \mathrm{Jy}$ (depending on the position in the field) could be detected. The VLBA detected one RQ AGN with a flux density $>400 \mu \mathrm{Jy}$, but the source was flagged only as reasonable ( $\mathrm{QF}=2$ in the catalogue of Bonzini et al. 2013).

Sources securely classified as RQ AGNs $(\mathrm{QF}=3)$ are fainter than $400 \mu \mathrm{Jy}$ (see Fig. 1). Deeper VLBI observations are therefore needed to probe the RQ AGN component in the ECDFS field to determine whether AGN cores are present. Here we report VLBI observations with the Australian Long Baseline Array (LBA), which is better suited for observations of this southern field.

VLBI observations at $\mu \mathrm{Jy}$ level are challenging. Here we focus on four RQ AGNs with secure classification $(\mathrm{QF}=3)$, selected from the brightest (with $200 \mu \mathrm{Jy}<\mathrm{S}_{1.4 \mathrm{GHz}}<400 \mu \mathrm{Jy}$ ) and unresolved (on arcsec-scale) sources, together with a control sample of four RL AGNs selected to match the flux density and redshift distribution of the RQ AGNs. This control sample not only allows us to check our data analysis procedure, but also measures the fraction of RL AGN detected in LBA observations.

\section{Observations and data reduction}

The LBA observations took place in five different runs, between March 2014 and March 2015, for a total of 51.5 h. Seven radio telescopes were requested for this experiment, with actual availability depending on the run (see Table 1). The data were correlated at the Pawsey Centre for Supercomputing.
Table 1. Observation runs of the project.

\begin{tabular}{ccccccc}
\hline \hline Run & Date & $\begin{array}{c}t_{\mathrm{Obs}} \\
(\mathrm{hrs})\end{array}$ & $\begin{array}{c}v_{\mathrm{Obs}} \\
(\mathrm{GHz})\end{array}$ & $\begin{array}{c}\mathrm{BW} \\
(\mathrm{MHz})\end{array}$ & Antennas & Target(s) \\
& & $(3)$ & $(4)$ & $(5)$ & $(6)$ & $(7)$ \\
\hline A & $09 / 03 / 2014$ & 9.5 & 1.666 & 64 & AK, AT, Cd, Ho, Mp, Pa & RQ26 \\
& & & & & & RL106 \\
& & & & & & RL728 \\
B & $04 / 06 / 2014$ & 11 & 1.650 & 32 & AT, Cd, Mp, Pa, Ti & RQ174 \\
& & & & & & RQ851 \\
C & $26 / 11 / 2014$ & 12 & 1.410 & 64 & AK, AT, Cd, Ho, Pa, Ti & RL183 \\
& & & & & & RL287 \\
D & $30 / 03 / 2015$ & 10 & 1.410 & 64 & AT, Cd, Ho, Mp, Pa & RQ851 \\
E & $31 / 03 / 2015$ & 9 & 1.410 & 64 & AT, Cd, Ho, Mp, Pa & RQ76 \\
\hline
\end{tabular}

Notes. $t_{\mathrm{Obs}}=$ Observation time; $v_{\mathrm{Obs}}=$ Average frequency of observation; $\mathrm{BW}=$ Total bandwidth; AK = ASKAP; AT = ATCA; Cd = Ceduna; Ho $=$ Hobart Mp = Mopra; Pa = Parkes; Ti = Tidbinbilla. Data from Run $\mathrm{C}$ were affected by technical problems, and were not used.

To maximise sensitivity, we observed in cycles of about six minutes by switching between target and phase calibrator (QSO J0348-2749, about 3.85 degrees away). Every 1-1.5 h, we observed the fringe finder (QSO B0208-512). When more than one target was observed, the six-minute cycles were shared between the targets, with the observing time inversely proportional to their flux density. Overall, $\sim 68 \%$ of the time was devoted to the target(s), $\sim 25 \%$ to the phase calibrator, and $\sim 7 \%$ to the fringe finder.

Calibration and imaging were carried out with the Astronomical Image Processing System ${ }^{1}$ (AIPS) software package. We first applied onsource and manual flagging using the task UVFLG. Ionospheric Faraday rotation and dispersive delay were corrected for using VLBATECR, and cross-correlation amplitude spectra were calibrated from auto-correlation data using ACCOR. We used CLCOR to correct the antenna gains and corrected for parallactic angle using the task VLBAPANG. We then corrected the instrumental phases and delays using the fringe finder and VLBAMPCL. Finally, we referred the phases of our targets to the phase of the phase calibrator through the task VLBAFRGP. When needed, a band-pass calibration was applied with the task BPASS. The a priori calibration was followed by a series of phase-only and amplitude+phase self-calibration loops on the phase calibrator, using the tasks IMAGR and CALIB. This solution was then used to calibrate the multi-data file.

We imaged the UV data with the task IMAGR into $2048 \times 2048$ pixel maps, with a pixel size of 1 mas and natural weighting. The images were cleaned with the Cotton-Schwab algorithm in a circle of 512 pixels radius centred on the detections. Typical restoring beams are $\sim 65 \times 42$ mas $^{2}$.

The maximum angular scale to which our LBA observations are sensitive is $\vartheta \sim 0.33^{\prime \prime}$ (A and B runs) and $\vartheta \sim 0.39^{\prime \prime}$ (D and E runs). In the redshift range of our targets $(1.0 \lesssim z \lesssim 2.9)$, this corresponds to a maximum linear scale of $\sim 2.7 \mathrm{kpc}$ (A and $\mathrm{B}$ runs) and $\sim 3.2 \mathrm{kpc}$ (D and E runs).

\section{Results}

Of the six sources successfully observed (two RL and four RQ AGNs), three were detected: one RL AGN (RL106) and two RQ AGNs (RQ851 and RQ76). The parameters of detected sources are listed in Table 2, while a detailed descriptions of the targets is given below.

\footnotetext{
1 http://www.aips.nrao . edu/index . shtml
} 

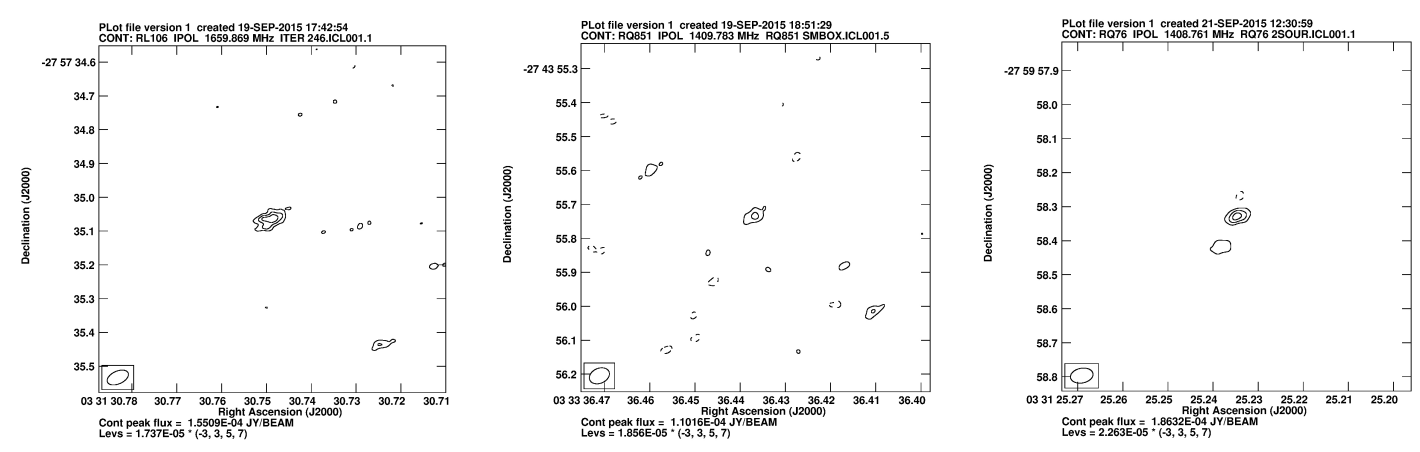

Fig. 2. VLBI-detected sources: RL 106 (left), RQ 851 (centre), and RQ76 (right). All the isophotes are drawn at $-3,3,5$ and 7 times the image $1 \sigma$ noise $\left(17.4,18.6\right.$ and $22.6 \mu \mathrm{Jy}$, respectively). All images are $\sim 1 \times 1 \operatorname{arcsec}^{2}$ wide.

\subsection{Detected sources}

RL106. Source 106 of Miller et al. (2013) is classified as a RL AGN by Bonzini et al. (2013) because of its high radio excess $\left(q_{24}\right.$ obs $=-0.99 \pm 0.18$; Lutz et al. 2011) and its location in the IRAC colour-colour diagram, where it lies well within the region occupied by elliptical galaxies $\left(\log \left(S_{5.8 \mu \mathrm{m}} / S_{3.6 \mu \mathrm{m}}\right)=-0.48 \pm 0.04\right.$; $\log \left(S_{8.0 \mu \mathrm{m}} / S_{4.5 \mu \mathrm{m}}\right)=-0.52 \pm 0.05 ; \quad$ Lonsdale et al. 2003; Surace et al. 2005). We found a VLBI counterpart (Fig. 2, left) coincident with the VLA position, with a peak flux density of $155 \mu \mathrm{Jy}(S / N \sim 9.1)$, or $\sim 43 \%$ of the $360 \mu \mathrm{Jy}$ measured by the VLA (Miller et al. 2013). The missing radio flux presumably represents resolved AGN structures, such as jets.

RQ851. Source 851 of Miller et al. (2013) is classified as a RQ AGN by Bonzini et al. (2013) because it has no radio excess $\left(q_{24 \mathrm{obs}}=0.34 \pm 0.03\right.$; Lonsdale et al. 2003; Surace et al. 2005) and is located in the AGN wedge of the IRAC colour-colour diagram $\left(\log \left(S_{5.8 \mu \mathrm{m}} / S_{3.6 \mu \mathrm{m}}\right)=\right.$ $0.26 \pm 0.02 ; \log \left(S_{8.0 \mu \mathrm{m}} / S_{4.5 \mu \mathrm{m}}\right)=0.14 \pm 0.01 ;$ Lonsdale et al. 2003; Surace et al. 2005). We detected a VLBI counterpart (Fig. 2, centre) $\sim 0.1^{\prime \prime}$ away from the VLA position, with a peak flux density of $110 \mu \mathrm{Jy}$, or $\sim 50 \%$ of the $222 \mu \mathrm{Jy}$ measured by the VLA (Miller et al. 2013). Our VLBI detection has a $S / N \sim 5.5$, and we therefore consider this detection as tentative.

RQ76. Source 76 of Miller et al. (2013) is classified as a RQ AGN by Bonzini et al. (2013) because it has no radio excess $\left(q_{24 \mathrm{obs}}=0.53 \pm 0.03 ;\right.$ Lonsdale et al. 2003; Surace et al. 2005), a powerful X-ray luminosity $\left(L_{2-8 \mathrm{keV}}=(4.2 \pm 0.3) \times 10^{44} \mathrm{erg} \mathrm{s}^{-1}\right.$; Lehmer et al. 2005), and is located in the AGN wedge of the IRAC colour-colour dia$\operatorname{gram}\left(\log \left(S_{5.8 \mu \mathrm{m}} / S_{3.6 \mu \mathrm{m}}\right)=0.50 \pm 0.03 ; \log \left(S_{8.0 \mu \mathrm{m}} / S_{4.5 \mu \mathrm{m}}\right)=\right.$ $0.60 \pm 0.02$; Lonsdale et al. 2003; Surace et al. 2005). We detected a VLBI counterpart (Fig. 2, right) $\sim 1.2^{\prime \prime}$ away from the VLA position, with a peak flux density of $186 \mu \mathrm{Jy}(S / N \sim 8.1)$, or $\sim 69 \%$ of the $269 \mu \mathrm{Jy}$ of the VLA flux density (Miller et al. 2013). The offset between the two radio positions is significant, but our detection lies inside the (large) positional error ellipse of the radio source $\left(2.9 \times 1.6 \operatorname{arcsec}^{2}\right)$ reported by Miller et al. (2013), is only $0.77^{\prime \prime}$ from the HST counterpart (Rix et al. 2004), and there is no other radio emission detected by the VLA, which is consistent with the measured VLBI detection. We therefore conclude that the VLBI detection is unambiguously the same source as the VLA detection, and the offset may be caused by extended emission, such as an AGN jet.

\subsection{Undetected sources}

$R Q 26$. Source 26 of Miller et al. (2013) is classified as a RQ AGN by Bonzini et al. (2013) because of its robust IR excess and location in the AGN wedge of the IRAC colour-colour diagram. We found no VLBI counterpart down to a $3 \sigma$ flux density level of $\sim 157 \mu \mathrm{Jy}$, or $\sim 50 \%$ of the VLA flux density of $318 \mu \mathrm{Jy}$ (Miller et al. 2013).

RL728. Source 728 of Miller et al. (2013) is classified as a RL AGN by Bonzini et al. (2013) because of a slight radio excess, strong X-ray emission, and location in the AGN wedge of the IRAC colour-colour diagram. We found no VLBI counterpart down to a $3 \sigma$ flux density level of $\sim 109 \mu \mathrm{Jy}$, or $\sim 30 \%$ of the VLA flux density of $326 \mu \mathrm{Jy}$ (Miller et al. 2013). This source is discussed in detail in Sect. 5.

$R Q 174$. Source 174 of Miller et al. (2013) is classified as a RQ AGN by Bonzini et al. (2013) because of a significant IR excess and high X-ray luminosity. We found no VLBI counterpart down to a $3 \sigma$ flux density level of $\sim 125 \mu \mathrm{Jy}$, or $\sim 42 \%$ of the VLA flux density of $300 \mu \mathrm{Jy}$ (Miller et al. 2013).

\section{Discussion and conclusions}

VLBI detections are usually considered as unambiguous evidence of AGN activity (Kewley et al. 2000). However, sensitivity improvements mean that VLBI can now detect a surface brightness of $T_{\mathrm{B}}<10^{5} \mathrm{~K}$, which can in principle have another origin. We therefore examined here whether this might be caused by compact H II regions, radio supernovae, or compact supernova remnants.

Compact H II regions are characterised at radio wavelengths by thermal free-free emission with $T_{\mathrm{B}} \sim 10^{4-5} \mathrm{~K}$ and sizes of a few pc, resulting in a radio luminosity $<10^{20} \mathrm{~W} \mathrm{~Hz}^{-1}$ (Hughes et al. 2007), far lower than the luminosities of our VLBI detected sources $\left(10^{23-25} \mathrm{~W} \mathrm{~Hz}^{-1}\right)$. To rule out the possibility that we detected bright supernova remnants or radio supernovae, we estimated the supernova rate corresponding to our radio luminosities. Following Kewley et al. (2000), we estimated the rate of radio-young supernovae $\left(v^{\mathrm{YSN}}\right)$ and of old supernova remnants $\left(v^{\mathrm{SNR}}\right)$ and list the result in Table 2 (Cols. 11 and 12). These values greatly exceed the rates for even the most extreme starburst galaxies $\left(\lessgtr 0.4 \mathrm{SN} \mathrm{yr}^{-1}\right.$; Mannucci et al. 2003). We are therefore confident that the emission we detect in our sources is due to AGN activity.

Recently, Bonzini et al. (2015) revisited the FIR SFR estimates of the ECDFS sample using the Herschel PACS data. This allowed them to obtain more reliable estimates of the source radio excess than those that were based on the $q_{24}$ parameter alone (Bonzini et al. 2013).

All the RQ AGNs we targeted lie below the radio/FIR correlation derived by Bonzini et al. (2015), presumably due to our bright flux limit $\left(S_{\mathrm{VLA}} \gtrsim 0.2 \mathrm{mJy}\right)$. On the other hand, the two LBA-detected RQ AGNs show radio excesses that are fully consistent with the overall distribution of the RQ AGN subsample around the correlation (i.e. they are not outliers). This 
Table 2. Summarised characteristics of our detections.

\begin{tabular}{|c|c|c|c|c|c|c|c|c|c|c|c|}
\hline Target & $\begin{array}{c}S_{\mathrm{VLBI}} \\
(\mu \mathrm{Jy}) \\
(2) \\
\end{array}$ & $\begin{array}{c}S_{\mathrm{VLBI}} / S_{\mathrm{VLA}} \\
(3) \\
\end{array}$ & $\begin{array}{c}\mathrm{rms} \\
(\mu \mathrm{Jy} / \mathrm{beam}) \\
(4)\end{array}$ & $\begin{array}{c}K \text {-corr. }^{a} L_{1.4 \mathrm{GHz}} \\
\left(\times 10^{23} \mathrm{~W} / \mathrm{Hz}\right) \\
(5)\end{array}$ & $\begin{array}{c}\text { Restoring beam } \\
\left(\mathrm{mas}^{2}\right) \\
(6)\end{array}$ & $\begin{array}{c}T_{\mathrm{B}} \\
\left(\times 10^{4} \mathrm{~K}\right) \\
(7)\end{array}$ & $\begin{array}{l}z \\
(8) \\
\end{array}$ & $\begin{array}{c}\text { Linear scale } \\
\text { (pc) } \\
(9) \\
\end{array}$ & $\begin{array}{c}\text { Host } \\
\text { type }^{e} \\
(10) \\
\end{array}$ & $\begin{array}{c}v^{\mathrm{YSN}} \\
\left(\mathrm{SN} \mathrm{yr}^{-1}\right) \\
(11)\end{array}$ & $\begin{array}{c}v^{\mathrm{SNR}} \\
\left(\mathrm{SN} \mathrm{yr}^{-1}\right) \\
(12)\end{array}$ \\
\hline RQ26 & $\$ 157$ & $\lessgtr 0.49$ & 52 & $\ldots$ & $\ldots$ & $\ldots$ & $1.59^{b}$ & $\ldots$ & - & $\ldots$ & $\ldots$ \\
\hline RL106 & $155 \pm 29$ & $0.43 \pm 0.08$ & 17 & $6.6 \pm 1.2$ & $\sim 67 \times 38$ & $2.7 \pm 0.5$ & $1.06^{c}$ & $\lesssim 544 \times 308$ & $\mathrm{P}$ & $\sim 126$ & $\sim 11$ \\
\hline RL728 & $\$ 109$ & $\lessgtr 0.33$ & 36 & $\ldots$ & $\ldots$ & $\ldots$ & $1.08^{b}$ & $\ldots$ & SB & $\ldots$ & $\ldots$ \\
\hline RQ174 & $\lessgtr 125$ & $\lessgtr 0.42$ & 42 & $\ldots$ & $\ldots$ & $\ldots$ & $2.85^{c}$ & $\ldots$ & MS & $\ldots$ & $\ldots$ \\
\hline RQ851 & $110 \pm 26$ & $0.50 \pm 0.12$ & 19 & $9.7 \pm 2.3$ & $\sim 62 \times 44$ & $2.5 \pm 0.6$ & $1.35^{d}$ & $\lessgtr 521 \times 370$ & MS & $\sim 163$ & $\sim 15$ \\
\hline RQ76 & $186 \pm 36$ & $0.69 \pm 0.14$ & 23 & $17.2 \pm 3.3$ & $\sim 67 \times 43$ & $4.0 \pm 0.8$ & $1.38^{b}$ & $\lessgtr 564 \times 362$ & SB & $\sim 290$ & $\sim 26$ \\
\hline
\end{tabular}

Notes. ${ }^{(a)}$ Rest-frame $1.4 \mathrm{GHz}$ luminosities were obtained by using measured 1.4-5 GHz spectral indices, based on data from Hales et al. (2014) and Huynh et al. (2012). For RQ851 and RQ76, $5 \mathrm{GHz}$ information is lacking, and we adopted the value of -0.75 (e.g., Kukula et al. 1998); ${ }^{(b)}$ spectroscopic, Silverman et al. (2010); ${ }^{(c)}$ photometric, Rafferty et al. (2011); (d) photometric, Taylor et al. (2009); ${ }^{(e)}$ from Bonzini et al. (2015): $\mathrm{P}=$ Passive; $\mathrm{SB}=$ starbust galaxy; $\mathrm{MS}$ = main sequence galaxy.

may indicate that compact radio cores are a common component of RQ AGNs, at least for those lying below the radio/FIR correlation. Bonzini et al. (2015) discussed the larger dispersion of RQ AGNs around the radio/FIR correlation with respect to the dispersion of SFGs and hypothesised that it might be due to contamination by AGN emission. Here we provide direct evidence that this is the case. When radio cores are removed, the two detected RQ AGNs fall nicely on the radio/FIR correlation. It is also interesting to note that our undetected RL AGN (RL728) is associated with an SB galaxy and lies close to the radio/FIR correlation, based on the new FIR SFR estimation of Bonzini et al. (2015). We can therefore argue that this is most likely a misclassified source.

To summarise, we found direct evidence that some of the AGNs classified as RQ in deep radio samples have mas-scale, AGN-triggered radio cores, at least at flux densities $S_{\text {VLA }} \gtrsim$ $0.2 \mathrm{mJy}$. Such cores can account for a significant fraction of the total radio emission (up to $\sim 70 \%$ in RQ76). Although limited in a statistical sense, our findings are supported by similar results by Herrera Ruiz et al. (2016), who found VLBI cores in RQ QSOs in the COSMOS field. Further support comes from the 12 RQQ sources (according to the classification of Bonzini et al. 2013) observed by Chi et al. (2013), four of which were detected with VLBI.

On the other hand, it is clear that at redshifts $z \gtrsim 1$ many RL AGNs are hosted by galaxies with strong star formation (Alexander et al. 2005; Daddi et al. 2007; Norris et al. 2007; Alexander 2008) and that these star-forming RL AGNs dominate the most radio-luminous AGNs at high redshift (Rees et al. 2016).

We therefore expect that the focus of future studies will shift from determining the nature of RQ sources to measuring the fraction of bolometric luminosity that is contributed by AGN and SF activities. However, further work is needed to understand the factors that determine whether a RQ AGN has a VLBI-detectable core.

Acknowledgements. The authors thank the anonymous referee for the valuable comments, that allowed us to improve the discussion of our results. A.M. is responsible for the content of this publication. A.M. acknowledges funding by a Cotutelle International Macquarie University Research Excellence Scholarship (iMQRES). I.P. and R.P.N. acknowledge support from the Ministry of Foreign Affairs and International Cooperation, Directorate General for the Country Promotion (Bilateral Grant Agreement ZA14GR02 - Mapping the Universe on the Pathway to SKA). The Australian SKA Pathfinder is part of the Australia Telescope National Facility which is managed by CSIRO. Operation of ASKAP is funded by the Australian Government with support from the National Collaborative Research Infrastructure Strategy. Establishment of the Murchison
Radio-astronomy Observatory was funded by the Australian Government and the Government of Western Australia. ASKAP uses advanced supercomputing resources at the Pawsey Supercomputing Centre. We acknowledge the Wajarri Yamatji people as the traditional owners of the Observatory site The Australia Telescope Compact Array (ATCA), the Parkes radio telescope, the Mopra radio telescope, and the Long Baseline Array, are part of the Australia Telescope National Facility which is funded by the Australian Government for operation as a National Facility managed by CSIRO. This work was supported by resources provided by the Pawsey Supercomputing Centre with funding from the Australian Government and the Government of Western Australia. This research has made use of NASA's Astrophysics Data System.

\section{References}

Alexander, D. M. 2008, Astron. Nachr., 329, 127

Alexander, D. M., Smail, I., Bauer, F. E., et al. 2005, Nature, 434, 738 Bonzini, M., Padovani, P., Mainieri, V., et al. 2013, MNRAS, 436, 3759 Bonzini, M., Mainieri, V., Padovani, P., et al. 2015, MNRAS, 453, 1079 Chi, S., Barthel, P. D., \& Garrett, M. A. 2013, A\&A, 550, A68

Daddi, E., Alexander, D. M., Dickinson, M., et al. 2007, ApJ, 670, 173 Del Moro, A., Alexander, D. M., Mullaney, J. R., et al. 2013, A\&A, 549, A59 Donley, J. L., Koekemoer, A. M., Brusa, M., et al. 2012, ApJ, 748, 142 Franzen, T. M. O., Banfield, J. K., Hales, C. A., et al. 2015, MNRAS, 453, 4020 Hales, C. A., Norris, R. P., Gaensler, B. M., et al. 2014, MNRAS, 441, 2555 Herrera Ruiz, N., Middelberg, E., Norris, R. P., \& Maini, A. 2016, A\&A, 589, L2

Hughes, A., Staveley-Smith, L., Kim, S., Wolleben, M., \& Filipović, M. 2007, MNRAS, 382, 543

Huynh, M. T., Hopkins, A. M., Lenc, E., et al. 2012, MNRAS, 426, 2342 Kewley, L. J., Heisler, C. A., Dopita, M. A., et al. 2000, ApJ, 530, 704

Kukula, M. J., Dunlop, J. S., Hughes, D. H., \& Rawlings, S. 1998, MNRAS, 297, 366

Lehmer, B. D., Brandt, W. N., Alexander, D. M., et al. 2005, ApJS, 161, 21

Lonsdale, C. J., Smith, H. E., Rowan-Robinson, M., et al. 2003, PASP, 115, 897

Lutz, D., Poglitsch, A., Altieri, B., et al. 2011, A\&A, 532, A90

Mannucci, F., Maiolino, R., Cresci, G., et al. 2003, A\&A, 401, 519

Middelberg, E., Deller, A., Morgan, J., et al. 2011, A\&A, 526, A74

Mignano, A., Prandoni, I., Gregorini, L., et al. 2008, A\&A, 477, 459

Miller, N. A., Bonzini, M., Fomalont, E. B., et al. 2013, ApJS, 205, 13

Norris, R. P., Middelberg, E., \& Boyle, B. J. 2007, in Deepest Astronomical

Surveys, eds. J. Afonso, H. C. Ferguson, B. Mobasher, \& R. Norris, ASP Conf. Ser., 380, 229

Padovani, P. 2011, MNRAS, 411, 1547

Rafferty, D. A., Brandt, W. N., Alexander, D. M., et al. 2011, ApJ, 742, 3

Rees, G. A., Spitler, L. R., Norris, R. P., et al. 2016, MNRAS, 455, 2731

Rix, H.-W., Barden, M., Beckwith, S. V. W., et al. 2004, ApJS, 152, 163

Roy, A. L., Norris, R. P., Kesteven, M. J., Troup, E. R., \& Reynolds, J. E. 1998, MNRAS, 301, 1019

Seymour, N., Dwelly, T., Moss, D., et al. 2008, MNRAS, 386, 1695

Silverman, J. D., Mainieri, V., Salvato, M., et al. 2010, ApJS, 191, 124

Smolčić, V., Padovani, P., Delhaize, J., et al. 2015, in Proc. Advancing Astrophysics with the Square Kilometre Array (AASKA14), PoS(AASKA14)069 Surace, J. A., Shupe, D. L., Fang, F., et al. 2005, BAAS, 37, 1246

Taylor, E. N., Franx, M., van Dokkum, P. G., et al. 2009, ApJS, 183, 295

Xue, Y. Q., Luo, B., Brandt, W. N., et al. 2011, ApJS, 195, 10 